\title{
Executive Functions in 5- to 8-Year Olds: Developmental Changes and Relationship to Academic Achievement
}

\author{
Marianne Röthlisberger ${ }^{1}$, Regula Neuenschwander ${ }^{1},{\text { Patrizia } \text { Cimeli }^{1} \& \text { Claudia M. Roebers }}^{1}$ \\ ${ }^{1}$ Department of Developmental Psychology, University of Bern, Switzerland \\ Correspondence: Marianne Röthlisberger, Department of Psychology, University of Bern, Fabrikstrasse 8, \\ CH-3000 Bern 12, Switzerland. Tel: 41-031-631-4182. E-mail: marianne.roethlisberger@psy.unibe.ch
}

Received: June 5, 2013

Accepted: September 24, 2013

Online Published: October 14, 2013

doi:10.5539/jedp.v3n2p153

URL: http://dx.doi.org/10.5539/jedp.v3n2p153

\begin{abstract}
Pronounced improvements in executive functions (EF) during preschool years have been documented in cross-sectional studies. However, longitudinal evidence on EF development during the transition to school and predictive associations between early EF and later school achievement are still scarce. This study examined developmental changes in EF across three time-points, the predictive value of EF for mathematical, reading and spelling skills and explored children's specific academic attainment as a function of early EF. Participants were 323 children following regular education; 160 children were enrolled in prekindergarten (younger cohort: 69 months) and 163 children in kindergarten (older cohort: 78.4 months) at the first assessment. Various tasks of EF were administered three times with an interval of one year each. Mathematical, reading and spelling skills were measured at the last assessment. Individual background characteristics such as vocabulary, non-verbal intelligence and socioeconomic status were included as control variables. In both cohorts, changes in EF were substantial; improvements in EF, however, were larger in preschoolers than school-aged children. EF assessed in preschool accounted for substantial variability in mathematical, reading and spelling achievement two years later, with low EF being especially associated with significant academic disadvantages in early school years. Given that EF continue to develop from preschool into primary school years and that starting with low EF is associated with lower school achievement, EF may be considered as a marker or risk for academic disabilities.
\end{abstract}

Keywords: developmental changes, executive functions, academic achievement, preschool and school children

\section{Introduction}

Research on EF in young children requires ongoing attention, given the association of EF with different aspects of child development and dysfunctional outcomes (Blair \& Razza, 2007; Hinshaw, Carte, Fan, Jassy, \& Owens; 2007; Hughes \& Ensor, 2007). An improved understanding of the role of EF-especially in young children-will facilitate the development of educational intervention programs that promote competence and resilience in children at risk of school failure. To address this challenge, longitudinal studies to inform developmental changes in early EF and investigations to explore the effect of EF on school outcomes are needed. The present two-year longitudinal study adds new aspects to questions about developmental changes in EF between preschool and school age and about the specific relationship between early EF and later mathematical, reading and spelling skills.

Broadly defined, EF encompass a set of cognitive processes that enable conscious control of thought toward goal-directed action in novel, complex and/or changing situations. In past research a multicomponent model of EF with inhibition, defined as the overriding of prepotent responses, working memory, defined as keeping and updating information in mind, and cognitive flexibility, defined as the shifting between mindsets, tasks or strategies has been suggested (Best \& Miller, 2010; Davidson, Amso, Anderson, \& Diamond, 2006; Garon, Bryson, \& Smith, 2008). Although there is evidence in favor of the componential view of EF (Lehto, Juujärvi, Kooistra, \& Pulkkinen, 2003, Miyake et al., 2000), some studies-particularly ones carried out in children-, failed to find this tripartite structure of EF (Lee et al., 2012; Van der Sluis, de Jong, \& Leij, 2007; Van der Ven, Kroesbergen, Boom, \& Lesemann, 2012). During early childhood, there is even evidence for a strong assumption of a unitary structure of EF (Hughes, Ensor, Wilson, \& Graham 2010; Wiebe, Espy, \& Charak, 2008; Wiebe et al. 2011; Willoughby, Blair, Wirth, Greenberg, \& Investigators, 2012a). 
Despite the large support of cross-sectional studies showing a pronounced improvement of EF over the preschool-age (Carlson, 2005; Davidson et al., 2006); studies examining developmental sequences within longitudinal designs are scarce (cf. Willoughby, Wirth, Blair, \& Investigators, 2012b). Moreover, there are even fewer longitudinal studies of EF between preschool- and school-age (Best \& Miller, 2010). One reason for this absence could be the difficulty of finding EF tasks that are child appropriate and sensitive for a relatively broad age range. Research efforts in recent years have led to the development of child-appropriate measurement tools (Carlson, 2005). However, a number of these tasks risk masking individual differences in EF performance because of a binary distribution (pass/fail distinction) or an unsuitable difficulty level (Willoughby et al., 2012a). This means that researchers interested in studying EF development in young children are still faced with measurement constraints, such as task complexity, variability in performance, scalability of scores, or capacity of attention (Best \& Miller, 2010).

Up to now, the few studies using a prospective longitudinal design show developmental changes of EF in preschool- and/or young school-aged children (Altemeier, Abbott, \& Berninger, 2008; Hughes et al., 2010; Roebers, Röthlisberger, Cimelli, Michel, \& Neuenschwander, 2011; Van der Ven et al. 2012, Willoughby et al., 2012b). Additionally, it seems that the magnitude of developmental change is larger in preschoolers than in school-aged children (Altemeier et al., 2008; Roebers et al., 2011; Willoughby et al., 2012b). Moreover, developmental differences are task-specific in some cases, meaning that they show little developmental changes in one task and large changes in another task (Van der Ven et al., 2012). Therefore, selecting tasks that measure $\mathrm{EF}$ at preschool and school-age equally well are needed. To address this aspect, in the present study we carefully chose EF tasks which can be used to explore developmental trajectories of EF from preschool to young school-aged children. From the perspective that an improved understanding of EF development-especially in young children, when EF develop rapidly-will facilitate the monitoring of interventions that promote competence and resilience in children at risk for school failure (Diamond, 2007; Willoughby et al., 2012a), the present study is of particular importance.

In addition to the developmental issue, some studies have recently focused on EF as significant predictors of individual differences in children's academic achievement (Agostino, Johnson, \& Pascual-Leone, 2010; Borella, Carretti, \& Pelegrina, 2010; Raghubar, Barnes, \& Hecht, 2010). During the past decades, developmental and educational researchers have increased their efforts to better delineate and understand factors influencing young children's progress in mathematics and literacy (Duncan et al., 2007). Among these efforts, longitudinal studies have targeted cognitive antecedents such as emergent literacy (letter recognition, phonemic awareness, spelling etc.) and numeracy skills (number recognition, magnitude understanding, counting etc.), demonstrating predictive power for the respective domains (Aunio, \& Niemivitra, 2010; Clark, Sheffield, Wiebe, \& Espy, 2013; Jordan, Glutting, \& Ramineni, 2010; Schneider, 2009). Beside this domain-specific focus, researchers have also placed great emphasis on more general cognitive processes that scaffold mathematical and literacy achievement (Bull, Espy, \& Wiebe, 2008). Of the processes distinguished up-to-date, EF seem to be particularly relevant. For example, working memory has been linked to mathematical skills (De Smedt et al., 2009; Passolunghi, Vercelloni, \& Schadee, 2007; Toll, Van der Ven, Kroesbergen, \& Van Luit, 2010; Van der Ven et al., 2012), reading skills (Christopher et al., 2012; Sesma, Mahone, Levine, Eason, \& Cutting, 2009) and spelling skills in school-aged children (Hooper et al., 2011). No comparable associations has been found between the other EF components-inhibition and cognitive flexibility- and mathematical, reading and spelling achievement, suggesting that working memory plays a more prominent role in academic skills at least in school-aged children (Altemeier et al., 2008; Lee et al., 2012; Toll et al., 2010; Van der Sluis, de Jong, \& van der Leij, 2007).

For preschooler and young school-age children, the relationship between EF and academic achievement is less clear. In addition to working memory, inhibition as well as cognitive flexibility are closely linked with performance on pre-math and pre-reading tasks, suggesting that in contrast to findings in school-aged children, the three EF components are of equal importance in early childhood (Bull et al., 2008; Clark, Pritchard, \& Woodward, 2010; Espy et al., 2004; Roebers et al., 2011; Willoughby et al., 2012). Together with the assumption of a more unitary structure of EF in preschool children, these results support research in which a composite measure of EF has been used and in which strong associations with math and reading achievement have been found (Clark et al., 2010; Welsh, Nix, Blair, Bierman, \& Nelson, 2010). Whether and to what extent $\mathrm{EF}$ are more strongly associated with math than with reading skills or vice versa is still being discussed. Some findings suggest a stronger link between EF and math skills (Brock, Rimm-Kaufman, Nathanson, \& Grimm, 2009; Willoughby et al., 2012a), while others do not, instead advocating for a domain-general association between EF and academic attainment (Best, Miller, \& Naglieri, 2011; Welsh et al., 2010). One explanation for the discrepancies in the literature could be the differences in EF tasks used (Booth, Boyle, \& Kelly, 2010). 
Additionally, it appears that EF are more closely linked to certain aspects of academic skills (i.e., maths: applied problems) than others (i.e., spelling) (Best et al., 2011).

Concerning the potential confounding factors that may account for the associations between EF and academic skills, recently some studies have been carried out in young children. They found that the observed associations can partly be explained by intelligence, socioeconomic status (SES), social network support and socio-affective functioning as well as socio-demographic characteristics (Bull, Espy, Wiebe, Sheffield, \& Nelson, 2011; Clark et al, 2010; Monette, Bigras, \& Guay, 2011; Navarro et al., 2012). Although there are few studies reporting significant effects of confounding variables, the above results underscore the need to examine them carefully.

In sum, the number of studies that explore the link between EF and academic achievement-even in preschool and young school children-is rapidly growing. However, to our knowledge these associations have never been investigated within the context of a prolonged longitudinal study in which a greater variability of change may provide a stronger test of the EF effect on academic achievement. Moreover, there is a lack of studies that include various tests of math, reading and spelling in order to qualify the impact of EF in different types of academic learning (Lee et al., 2012). While most of the existing studies realized an individual differences approach, we additionally applied a group comparison in which we contrast children with high and medium, versus low EF longitudinally and prospectively. This way it also becomes possible to estimate the impact of EF for academic achievement in absolute terms. Assuming that an academic achievement gap progressively widens throughout the first school years, the early identification of children at risk by means of potential predictors-such as EF-is crucial (Passolunghi \& Lanfranchi, 2012; Toll et al., 2010).

\subsection{Study Aims}

The purpose of our study was threefold. First, we tried to monitor developmental changes of different EF tasks in prekindergarten, kindergarten and early school children using a longitudinal design. Given that EF development in young children is especially pronounced, we hypothesized that performances on all three EF tasks would increase during the whole period of time and that developmental changes would be larger in preschoolers than school-aged children. Second, we sought to determine the role of preschool EF in predicting mathematical, reading and spelling skills at the end of Grade 1 and Grade 2, and to verify whether the association remained when confounding variables such as vocabulary, nonverbal IQ, and SES were controlled for. Based on existing studies, we assumed that EF measured at preschool years are linked to mathematical, reading and spelling achievement two years later. As we decided to analyze the prediction of EF on task level, our current question was whether the relationship of EF with school achievement is consistent across achievement subtest and cohorts. Third, we aimed to explore whether early EF would have different impacts on various academic tests and whether children with low, medium and high EF performance in preschool years would show different achievement patterns in school later on. This last question was particularly emphasized, assuming that an early detection of EF deficits may be helpful in order to implement educational interventions for at risk children and preparing them for school.

\section{Method}

\subsection{Participants}

All children included in the analyses were selected from a large data set $(N=446$ children) used to investigate both typical and atypical development during transition to school. The present study focused on typically developing children following regular transition to school. As there are studies showing EF deficits in children with special educational needs as well as schooling effects on EF development (Burrage et al., 2008; Diamond et al. 2007; Lillard \& Else-Quest, 2006; Michel \& Roebers, 2008), children enrolled in special educational need classes, and children with grade retention or grade acceleration were excluded. Therefore, the sample consisted of 160 children attending prekindergarten at the first assessment (younger cohort; mean age 69.0 months, $S D=$ 4.4; range 61-79 months; $47 \%$ female) and 163 children attending kindergarten at the first assessment (older cohort; mean age: 78.4 months; $S D=4.0$; range $64-87$ months; $52 \%$ female). At the entry of the study, participants were enrolled in 47 classes distributed across 24 school areas in different urban and rural regions; because of grade progression, at the end of the study, the children were distributed across 75 classes in 48 schools. Parent's highest educational level was representative of the Swiss population (mother/ father: academic degree: $9.6 \% / 16 \%$; diploma of applied science: $12.7 \% / 30.2 \%$; vocational training: $59.6 \% / 39.3 \%$; university entrance diploma: $8.9 \% / 7.3 \%$; compulsory education: $9.2 \% / 7.3 \%)$. No significant differences between the prekindergarten and kindergarten children in terms of their socioeconomic background were found. The percentage of children with migration background was $19.8 \%$, a proportion that is representative for the Swiss 
population as well. It was verified that all children were able to speak the official language with sufficient fluency. Parental informed consent was obtained for all children prior to participation.

\subsection{Procedure}

Child assessments were conducted by trained examiners at three time points with 1-year intervals (from now on referred to as T1, T2, T3). For the younger cohort: at the end of prekindergarten year (T1), at the end of kindergarten year (T2), and at the end of Grade 1(T3); for the older cohort: at the end of kindergarten year (T1), at the end of Grade 1 (T2), and the end of Grade 2 (T3). EF measures were collected at each time point, while individual background characteristics were assessed at $\mathrm{T} 1$ and academic achievement at $\mathrm{T} 3$. To measure EF and individual background characteristics, children were tested individually in a separate room at their school during two 30-min sessions; the order of the tasks was counterbalanced. The testing of academic achievement was carried out in small groups in school classrooms. Children received small gifts for participating. Parents were asked to fill out a sociodemographic questionnaire.

\subsection{Measures}

Executive functions were measured using three tasks, partially programmed and run using E-Prime software (Psychology Software Tools PST; Pittsburgh, PA) and a composite score of EF were built. Individual background characteristics-vocabulary, non-verbal IQ, SES-which are known to be related to EF and academic achievements were included as control variables. Academic achievement in the domains of mathematics, reading and spelling were measured with standardized school achievement tests. Means and standard deviations of the test scores at $\mathrm{T} 1$ and $\mathrm{T} 3$ are shown in Table 1.

Table 1. Descriptive statistics

\begin{tabular}{lcccc}
\hline & \multicolumn{2}{c}{ Younger cohort $(N=160)$} & \multicolumn{2}{c}{ Older cohort $(N=163)$} \\
& $M(S D)$ & Range & $M(S D)$ & Range \\
\hline Time 1 & & & & \\
EF (composite z-score) & $.03(.7)$ & $-1.7-1.4$ & $.03(.6)$ & $-2.0-1.3$ \\
Intelligence & $5.0(2.0)$ & $0-11$ & $6.6(2.0)$ & $2-12$ \\
Vocabulary & $41.7(6.4)$ & $20-51$ & $46.6(5.4)$ & $14-55$ \\
SES & $8.9(3.3)$ & $4-19$ & $9.6(3.3)$ & $3-18$ \\
Time 3 & & & \\
Mathematics & & & & \\
Magnitude comparison & $8.1(3.1)$ & $1-12$ & $9.3(2.7)$ & $1-12$ \\
Equation & $13.1(5.9)$ & $-4.0-27.0$ & $20.1(5.7)$ & $-1.5-34.0$ \\
Sequences of numbers & $8.4(3.4)$ & $0-16$ & $11.9(2.6)$ & $0-17$ \\
Arithmetic & $4.8(2.2)$ & $0-12$ & $7.9(2.5)$ & $2-12$ \\
Literacy & & & & \\
Reading speed & $42.7(19.8)$ & $5-116$ & $70.2(22.6)$ & $0-120$ \\
Reading comprehension & $12.7(8.9)$ & $0-45$ & $25.0(9.9)$ & $6-55$ \\
Spelling & $117.1(16.4)$ & $14-139$ & $130.4(5.0)$ & $115-141$ \\
\hline
\end{tabular}

\subsubsection{Inhibition}

Inhibitory performance was assessed with an adapted version of the Fruit Stroop task by Archibald \& Kerns (1999) In the Fruit Stroop task (Röthlisberger, Neuenschwander, Michel, \& Roebers, 2010; Loher \& Roebers, 2013), children were presented pictures of different fruits and vegetables, either in correct, black and white or incorrect colour. Four different pages, with five rows of five stimuli in each row were presented. On the first page, colored squares, and on the second page, colored fruit and vegetables were displayed. The child was asked to name the colors of the squares and fruits/vegetables as quickly as possible. The third page consisted of the same fruits/vegetables, however, printed in black and white. In the final condition, the same fruits/vegetables 
were displayed in incongruent colors (e.g., blue banana) and the child's task was to name the original color of the fruit/vegetables. The following interference score with time for completion was used as dependent variable: (page 4) - [(page 1*page 3) / (page 1 + page 3)] (Archibald \& Kerns, 1999). Smaller scores were interpreted as reflecting higher abilities in resistance to stimulus-incompatibility. The Fruit Stroop task showed good reliability (Split-half reliability at $\mathrm{T} 1 ; \mathrm{r}=.78$ ) and appropriate validity (significant differences of scores between children with and without special educational needs).

\subsubsection{Working Memory}

To measure working memory capacity, a task which is analogous to the backwards digit span task was used: Backwards Color Recall task (Schmid, Zoelch, \& Roebers, 2008). Children were presented sequences of colored discs (1 disc per s) on a computer screen and asked to recall the sequence in the reverse order. The task started with a two-disc sequence. Sequence length was increased by one disc when the child correctly recalled two of three sequences on a particular level. The number of trials recalled correctly was used as the final score. TestRetest reliability in a sample of 4-5 years old was $r=.57$ (Schmid et al. 2008), which represents an equal reliability coefficient as has been shown for the Working Memory Test Battery for Children (WMTC-C; Pickering \& Gathercole, 2001). Based on the large data set, the Backwards Color Recall task showed appropriate validity (significant differences of scores between children with and without special educational needs).

\subsubsection{Cognitive Flexibility}

In the computerized Cognitive Flexibility task (Roebers \& Kauer, 2009), two categories of fish (introduced as two fish families) were presented simultaneously on the right and left side of a computer screen. Children were told to feed the two families of fish consecutively (i.e., to feed a member of one family in the first and a member of the other family in the next trial). Fish were fed by pressing a button on the side corresponding to the appearance of the fish (right or left). The proportion of correct responses, collapsed across switch and non-switch trials were used as the dependent variable. The Cognitive Flexibility task demonstrated good reliability (Split-half reliability at $\mathrm{T} 1 ; \mathrm{r}=.64$ ) and appropriate validity (significant differences of scores between children with and without special educational needs).

\subsubsection{Composite Score of EF}

To account for the task impurity problem of any EF task and to reduce data and in an attempt to create a reliable composite score of children's overall EF at T1, a principal-component analysis was carried out. Significant correlations among the three EF measures ranged from $r=.29$, to $r=.37$. Results suggested that these measures were reflective of a single construct, accounting for $55 \%$ of the total variance across the measures (all factors loadings $>$.7). Therefore z-scores of all three EF measures were averaged to form an overall EF composite score.

\subsubsection{Vocabulary}

For the assessment of active and passive vocabulary, the Hannover-Wechsler Intelligence Scale for preschool children (HAWIVA-III; Ricken, Fritz, Schuck, \& Preuss, 2007) was applied. Raw scores of both tasks were averaged and used as the dependent variable.

\subsubsection{Non-Verbal Intelligence}

As a second control variable, non-nonverbal intelligence was measured with the Classification subtest from the Culture Fair Intelligence Scale designed for children aged 5 and up (CFT-1; Cattell, Weiss \& Osterland, 1997; test-retest reliability: .80). The number of correct items within the time limit was used as the dependent variable.

\subsubsection{Socioeconomic Background (SES)}

SES was assessed with a sociodemographic questionnaire: Parents were asked about their professional education and their current job situation. Both variables were coded with the Wegener Prestige Scale (Wegener, 1988) and used to calculate an index for the SES of the participating children.

\subsubsection{Mathematics}

Mathematical skills were measured using four subtests (used from standardized, curriculum-based achievement tests: TEDI-Math; Kaufmann et al., 200and HRT 1-4; Haffner, Baro, Parzer, \& Resch, 2005). In the Magnitude Comparison test, children had to select the field with the larger number of dots out of two different fields of dots. The Equation test consisted of 40 items in which quantities (e.g., $2+9<20$ ) had to be compared. In the Sequences test, children were presented with 20 sequences of numbers and had to continue these sequences (e.g., $5,1,6,2, \ldots)$. In the Arithmetic test, children were given 12 arithmetic problems containing two or more operations (e.g., 15-3-3+7=). For all tests, the number of correct solutions within the given time limit was used 
as the dependent variable. Performance on these subtests and children's performance in mathematics rated by their teachers were significantly correlated $(\mathrm{r}=.48$ to $\mathrm{r}=.51)$

\subsubsection{Literacy}

To assess literacy skills, three well established and standardized curriculum-based school achievement tests were administered. In the Reading speed tests, children were asked to search for the picture that matched the written word within the given time limit (WLLP; Küspert \& Schneider, 1998). In the Reading comprehension tests, children were asked to judge the correctness of different sentences within the given time limit (SLS; Mayringer \& Wimmer, 2003). Spelling skills were measured using a test in which children had to write down 22 words and a sentence which were read aloud (HSP; May, 2002). The number of correct graphemes (in words \& sentence) was used as the dependent measure. Performance on these subtests and children's performance in reading and spelling skills rated by their teachers correlated significantly $(r=.49$ to $r=.51)$.

\section{Results}

\subsection{Statistics and Data Analyses}

Individual scores below three standard deviations of the mean, based on the corresponding cohort, were considered as outliers and therefore removed. In addition, some data points were missing, either because the child refused to participate in a particular task, or due to computer difficulties or time constraints. The number of missing values never exceeded 3\% and can be inferred from the varying degrees of freedom reported in the analyses below.

Analyses were carried out in three stages: To address the issue of developmental changes in EF measures mixed ANOVAs with cohort (younger and older cohort) as the between-subject factor, and time point (T1, T2, T3) as within-subject factor were carried out. Following this, linear regression models were run to determine the net contributions of children's EF (T1) to the prediction of mathematical, reading or spelling skills two years later (T3) after controlling for SES, nonverbal IQ, vocabulary. Finally, in order to determine whether an assessment of EF in preschool years would provide insight which children may be affected by mathematical, reading or spelling difficulties later on, children were divided into three groups based on their overall EF composite score at T1. In the literature, different cut-off scores are proposed (i.e., Clark et al., 2010;-/+1SD of the mean). In order to ensure that in each group at least 10 children were classified, following cut-off points were used: Children who performed at least $0.8 S D$ below the group mean (younger cohort: $n=21$; older cohort: $n=16$ ) were classified as showing low EF, those who performed within $0.8 S D$ of the group mean (younger cohort: $n=115$; older cohort: $n=128$ ) were classified as showing medium EF and those who performed at least $0.8 S D$ above the group mean (younger cohort: $n=24$; older cohort: $n=19$ ) were classified as showing high EF. Separate ANCOVAs for the younger and older cohort with following covariates: SES, nonverbal IQ, and vocabulary skills were carried out for each academic achievement test separately. In case of a significant or marginally significant EF performance group effect, planned comparisons were conducted in which the medium and high EF performance groups were compared with the low EF performance group.

Significant correlations between EF composite scores at $\mathrm{T} 1$ and mathematical tests at $\mathrm{T} 3$ ranged from .30 to .49 (with one exception: in the older cohort, there was no significant correlations between the Magnitude Comparison test and the EF composite score, $r=.15$ ); the correlations between EF composite score at $\mathrm{T} 1$ and literacy tests at $\mathrm{T} 3$ were all significant and ranged from .24 to .40. For the EF composite score, the stability coefficients for the younger cohort were .55 and .61 for the one-year periods, and .45 for the two-year period. Analyses of the older cohort showed significant stability coefficients of .58 and .63 for the one-year periods, and .57 for the two-year period.

\subsection{Developmental Changes}

As can be seen in Figure 1, a continuous improvement of EF performance from T1 to T3 was found in both age groups. The mixed ANOVA's revealed a significant main effects of time for all EF measures: $F_{\text {Inhibition }}(2,632)=$ $244.4, p<.001, \eta_{\mathrm{p}}{ }^{2}=.44 ; F_{\text {Working memory }}(2,638)=152.8, p<.001, \eta_{\mathrm{p}}{ }^{2}=.32 ; F_{\text {Cognitive Flexibility }}(2,630)=296.2, p$ $<.001, \eta_{\mathrm{p}}{ }^{2}=.49$, and a main effect of cohort: $F_{\text {Inhibition }}(1,316)=70.3, p<.001, \eta_{\mathrm{p}}{ }^{2}=.18 ; F_{\text {Working memory }}(1,319)$ $=62.1, p<.001, \eta_{\mathrm{p}}{ }^{2}=.16 ; F_{\text {Cognitive Flexibility }}(1,315)=72.6, p<.001, \eta_{\mathrm{p}}{ }^{2}=.19$. Additionally, significant interaction effects between time and cohort were found for two EF measures: $F_{\text {Inhibition }}(2,632)=8.6, p<.001$, $\eta_{\mathrm{p}}^{2}=.03$; and $F_{\text {Working memory }}(2,638)=6.3, p<.01, \eta_{\mathrm{p}}{ }^{2}=.02$.

In addition, to explore and compare EF developmental changes in preschool and in school children, EF changes in children during the transition to school were omitted. The one-way ANOVA's demonstrated statistically significant differences between changes in preschool (from pre-kindergarten to kindergarten) and school children 
(from grade 1 to grade 2 ), $F_{\text {Inhibition }}(1,317)=8.7, p<.01, \eta_{\mathrm{p}}{ }^{2}=.03, F_{\text {Working memory }}(1,319)=17.3, p<.001, \eta_{\mathrm{p}}{ }^{2}$ $=.05, F_{\text {Cognitive Flexibility }}(1,317)=14.4, p<.001, \eta_{\mathrm{p}}{ }^{2}=.04$, yielding larger improvements of EF in favor of preschool children.
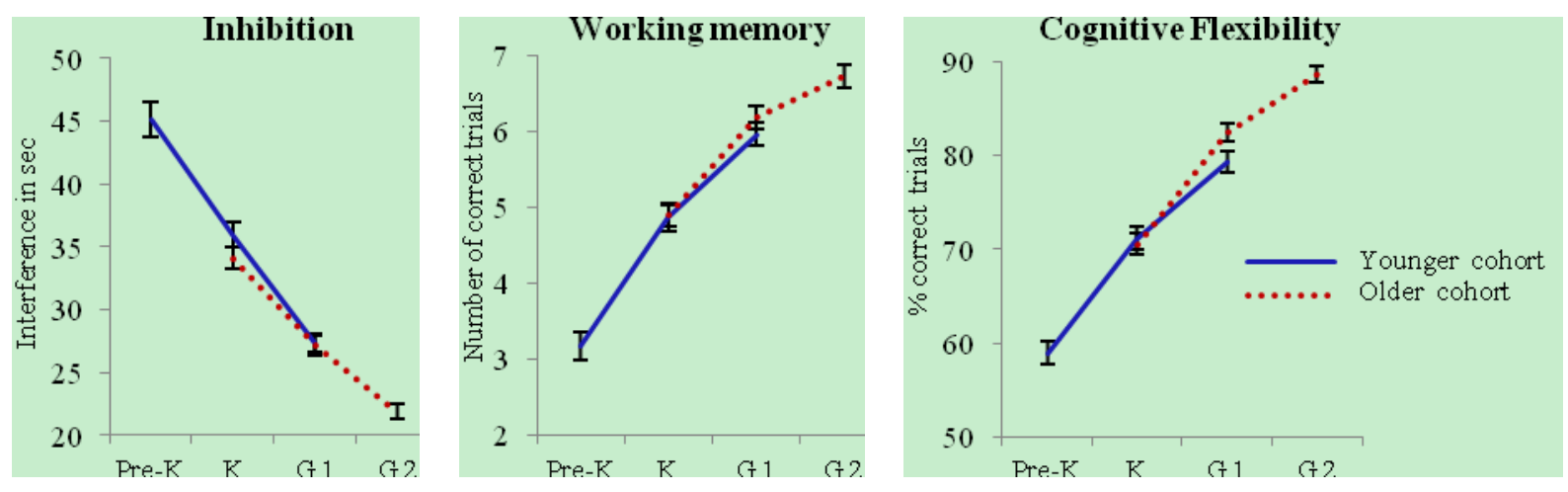

Figure 1. Developmental changes of Inhibition, Working memory and Cognitive Flexibility

Note. Pre-K: Pre-Kindergarten; K: Kindergarten; G 1: Grade 1; G 2: Grade 2. Error bars represent standard errors of the mean.

\subsection{Prediction of Academic Skills}

To assess the prediction of early EF for the different mathematical, reading and spelling tests assessed two years later, hierarchical multiple regression analyses were calculated for both cohorts separately. In the regression models, well-established correlates of academic achievement such as SES, nonverbal IQ and vocabulary were first entered as control variables, and the composite score of EF was entered thereafter to estimate the predictive power of EF over and above these background variables (see Table 2 and 3).

Mathematics: As shown in Table 2, when the control variables were accounted for, prekindergarten (younger cohort) and kindergarten (older cohort) children`s EF still explained an additional amount of variance in different mathematical sub-domains two years later. Interestingly in the older cohort, no significant unique variance was explained by early EF in the Magnitude comparison subtest.

Younger cohort: Magnitude comparison: $R^{2}=.10, \Delta R^{2}=.06, F(1,155)=10.30, p<.01$; Equation: $R^{2}=.23, \Delta$ $R^{2}=.13, F(1,155)=25.10, p<.001$; Sequences of numbers: $R^{2}=.20, \Delta R^{2}=.12, F(1,155)=23.64, p<.001$; Arithmetic: $R^{2}=.14, \Delta R^{2}=.06, F(1,155)=10.74, p<.01$.

Older cohort: Equation: $R^{2}=.24, \Delta R^{2}=.11, F(1,158)=22.96, p<.001$; Sequences of numbers: $R^{2}=.25, \Delta R^{2}$ $=.20, F(1,158)=41.93, p<.001$; Arithmetic: $R^{2}=.18, \Delta R^{2}=.08, F(1,158)=15.86, p<.01$.

Table 2. Hierarchical multiple regression analyses predicting different mathematic skills (T3) from early EF (T1)

\begin{tabular}{|c|c|c|c|c|c|c|c|c|c|c|c|c|c|c|c|c|}
\hline \multirow[b]{3}{*}{ Predictor } & \multicolumn{8}{|c|}{ Mathematics: Younger cohort } & \multicolumn{8}{|c|}{ Mathematics: Older cohort } \\
\hline & \multicolumn{2}{|c|}{$\begin{array}{l}\text { Magnitude } \\
\text { comparison }\end{array}$} & \multicolumn{2}{|c|}{ Equation } & \multicolumn{2}{|c|}{$\begin{array}{l}\text { Sequences of } \\
\text { numbers }\end{array}$} & \multicolumn{2}{|c|}{ Arithmetic } & \multicolumn{2}{|c|}{$\begin{array}{l}\text { Magnitude } \\
\text { comparison }\end{array}$} & \multicolumn{2}{|c|}{ Equation } & \multicolumn{2}{|c|}{$\begin{array}{l}\text { Sequences of } \\
\text { numbers }\end{array}$} & \multicolumn{2}{|c|}{ Arithmetic } \\
\hline & $\Delta R^{2}$ & $\beta$ & $\Delta R^{2}$ & $\beta$ & $\Delta R^{2}$ & $\beta$ & $\Delta R^{2}$ & $\beta$ & $\Delta R^{2}$ & $\beta$ & $\Delta R^{2}$ & $\beta$ & $\Delta R^{2}$ & $\beta$ & $\Delta R^{2}$ & $\beta$ \\
\hline Step 1 & $.04^{\dagger}$ & & $.10^{* *}$ & & $.08^{* *}$ & & $.08^{* *}$ & & $.08^{* *}$ & & $.13^{* *}$ & & $.05^{*}$ & & $.10^{* *}$ & \\
\hline \multicolumn{17}{|l|}{$\begin{array}{l}\text { Control } \\
\text { variables }^{\mathrm{a}}\end{array}$} \\
\hline Step 2 & $.06^{* *}$ & & $.13^{* * *}$ & & $.12^{* * *}$ & & $.06^{* *}$ & & .01 & & $.11^{* *}$ & & $.20^{* * *}$ & & $.08^{* *}$ & \\
\hline EF & & $.26^{* *}$ & & $.37^{* * *}$ & & $.37^{* * *}$ & & $.26^{* *}$ & & .11 & & $.35^{* * *}$ & & $.48^{* * *}$ & & $.31^{* * *}$ \\
\hline Total $R^{2}$ & $.10^{* *}$ & & $.23^{* * *}$ & & $.20^{* * *}$ & & $.14^{* * *}$ & & $.09^{* *}$ & & $.24^{* * *}$ & & $.25^{* * *}$ & & $.18^{* * *}$ & \\
\hline$n$ & 160 & & 160 & & 160 & & 160 & & 163 & & 163 & & 163 & & 163 & \\
\hline
\end{tabular}


Literacy: As shown in Table 3, when the control variables were accounted for, prekindergarten (younger cohort) and kindergarten (younger cohort) children's EF still explained additional variance in the different aspects of literacy skills two years later.

Younger cohort: Reading speed: $R^{2}=.18, \Delta R^{2}=.08, F(1,155)=15.49, p<.001$; Reading comprehension: $R^{2}$ $=.20, \Delta R^{2}=.07, F(1,155)=13.49, p<.001$; Spelling: $R^{2}=.12, \Delta R^{2}=.05, F(1,155)=8.94, p<.01$.

Older cohort: Reading speed: $R^{2}=.24, \Delta R^{2}=.11, F(1,158)=22.35, p<.001$; Reading comprehension: $R^{2}$ $=.23, \Delta R^{2}=.12, F(1,158)=24.53, p<.001$; Spelling: $R^{2}=.17, \Delta R^{2}=.04, F(1,158)=7.13, p<.01$.

Table 3. Hierarchical multiple regression analyses predicting different literacy skills (T3) from early EF (T1)

\begin{tabular}{|c|c|c|c|c|c|c|c|c|c|c|c|c|}
\hline \multirow[b]{3}{*}{ Predictor } & \multicolumn{6}{|c|}{ Literacy: Younger cohort } & \multicolumn{6}{|c|}{ Literacy: Older cohort } \\
\hline & \multicolumn{2}{|c|}{ Reading speed } & \multicolumn{2}{|c|}{$\begin{array}{c}\text { Reading } \\
\text { comprehension }\end{array}$} & \multicolumn{2}{|c|}{ Spelling } & \multicolumn{2}{|c|}{ Reading speed } & \multicolumn{2}{|c|}{$\begin{array}{c}\text { Reading } \\
\text { comprehension }\end{array}$} & \multicolumn{2}{|c|}{ Spelling } \\
\hline & $\Delta R^{2}$ & $\beta$ & $\Delta R^{2}$ & $\beta$ & $\Delta R^{2}$ & $\beta$ & $\Delta R^{2}$ & $\beta$ & $\Delta R^{2}$ & $\beta$ & $\Delta R^{2}$ & $\beta$ \\
\hline Step 1 & $.10^{* *}$ & & $.13^{* * *}$ & & $.07^{* *}$ & & $.13^{* * *}$ & & $.11^{* * *}$ & & $.13^{* * *}$ & \\
\hline \multicolumn{13}{|c|}{ Control variables ${ }^{\mathrm{a}}$} \\
\hline Step 2 & $.08^{* * *}$ & & $.07^{* * *}$ & & $.05^{* *}$ & & $.11^{* * *}$ & & $.12^{* * *}$ & & $.04^{* *}$ & \\
\hline $\mathrm{EF}$ & & $.31^{* * *}$ & & $.28^{* * *}$ & & & & $.35^{* * *}$ & & $.37^{* * *}$ & & $.21^{* * *}$ \\
\hline Total $R^{2}$ & $.18^{* * *}$ & & $.20^{* * *}$ & & $.12^{* * *}$ & & $.24^{* * *}$ & & $.23^{* * *}$ & & $.17^{* * *}$ & \\
\hline$n$ & 160 & & 160 & & 160 & & 163 & & 163 & & 163 & \\
\hline
\end{tabular}

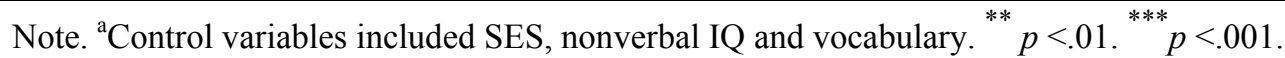

\subsection{Academic Profiles in Relationship with Early EF}

In order to examine specific academic achievement profiles of children with low, medium and high EF (composite score at T1), ANCOVAs with SES, nonverbal IQ and vocabulary skills as covariates were carried out for each single achievement test at T3 (see Figure 2 and 3). Only covariates that reached statistically significant levels are mentioned below.
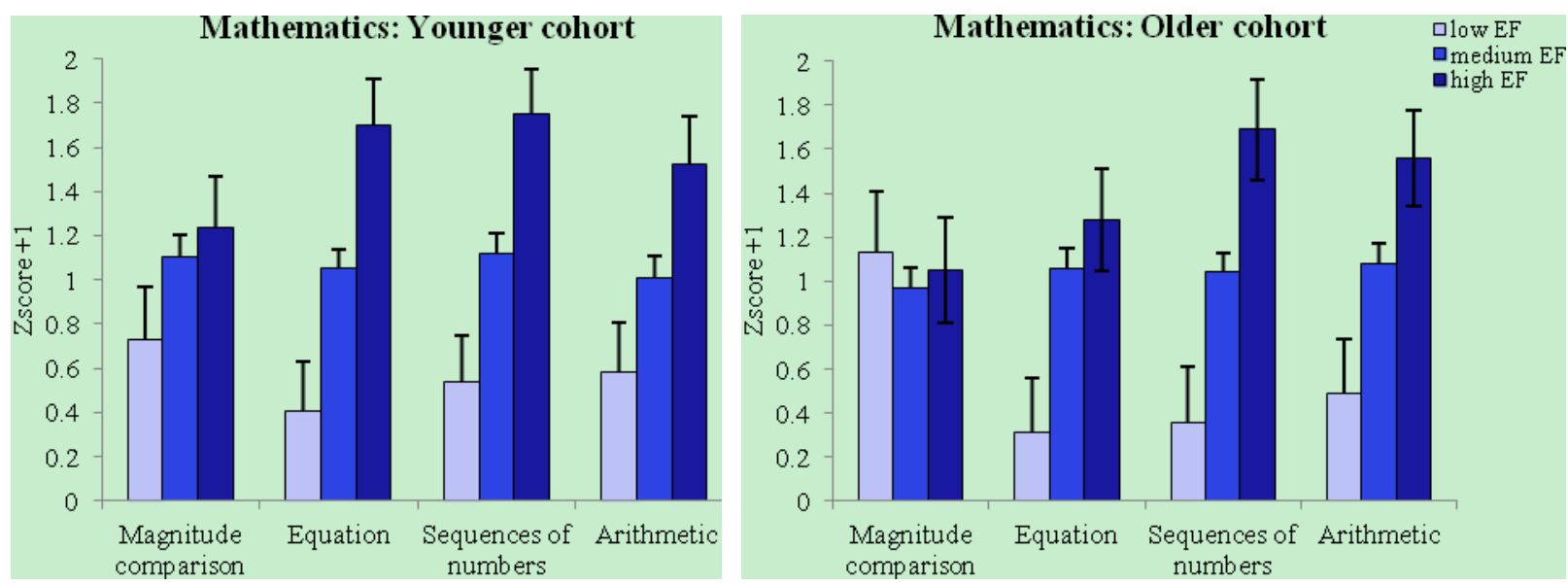

Figure 2. Mathematical achievement at Time 3 as a function of initial EF performance

Note. Bars represent estimated marginal means with SES, nonverbal IQ, and vocabulary as covariates and error bars represent standard errors of the mean. 


\section{Mathematics}

Younger cohort: There were significant effects of EF performance group (low, medium and high) in the Equation test $F(2,133)=8.7, p<.001, \eta_{\mathrm{p}}{ }^{2}=.12$, the Sequences test, $F(2,133)=8.5, p<.001, \eta_{\mathrm{p}}{ }^{2}=.11$, and the Arithmetic test, $F(2,133)=4.3, p<.05, \eta_{\mathrm{p}}{ }^{2}=.06$, even after controlling for the significant effect of the covariate SES, $F(1,133)=4.7, p<.05, \eta_{\mathrm{p}}{ }^{2}=.03$. For the Magnitude Comparison test, no significant effects of EF performance group were found. Planned contrasts revealed that children with medium or high EF showed significantly higher performance in the Equation test (medium EF: $\eta_{\mathrm{p}}{ }^{2}=.05$; high EF $\eta_{\mathrm{p}}{ }^{2}=.12$ ), and the Sequences test (medium EF: $\eta_{\mathrm{p}}{ }^{2}=.05$; high: $\mathrm{EF} \eta_{\mathrm{p}}{ }^{2}=.11$ ) compared to low EF at T1. For the Arithmetic test, only children with high EF differed significantly from children with low $\mathrm{EF}\left(\eta_{\mathrm{p}}{ }^{2}=.06\right)$.

Older cohort: There were significant effects of EF performance group (low, medium and high) in the Equation test $F(2,139)=4.4, p<.05, \eta_{\mathrm{p}}{ }^{2}=.06$, even after controlling for the significant effect of the covariate of nonverbal IQ $F(1,139)=6.6, p<.05, \eta_{\mathrm{p}}{ }^{2}=.05$. There were also significant effects of EF performance group (low, medium and high) in the Sequences test, $F(2,139)=7.2, p<.01, \eta_{\mathrm{p}}{ }^{2}=.09$, and the Arithmetic test, $F(2$, $139)=4.8, p<.05, \eta_{\mathrm{p}}{ }^{2}=.06$. Planned contrasts revealed that children with medium or high EF showed significantly higher performance in the Equation test (medium EF: $\eta_{\mathrm{p}}{ }^{2}=.05$; high EF: $\eta_{\mathrm{p}}{ }^{2}=.05$ ), in the Sequences test (medium EF: $\eta_{\mathrm{p}}{ }^{2}=.05$; high EF: $\eta_{\mathrm{p}}{ }^{2}=.09$ ) and the Arithmetic test (medium EF: $\eta_{\mathrm{p}}{ }^{2}=.04$; high $\left.\mathrm{EF}: \eta_{\mathrm{p}}{ }^{2}=.06\right)$ compared to children with low EF at T1.
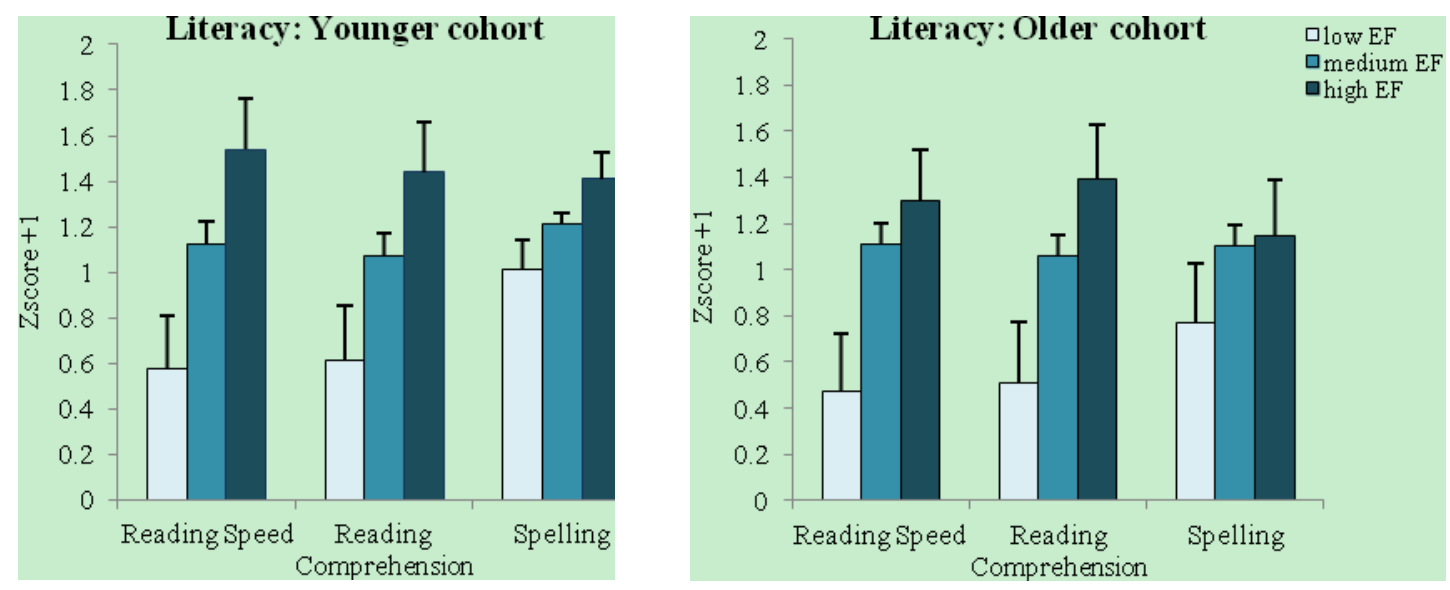

Figure 3. Literacy achievement at Time 3 as a function of initial EF performance

Note. Bars represent estimated marginal means with SES, nonverbal IQ, and vocabulary as covariates and error bars represent standard errors of the mean.

\section{Literacy}

Younger cohort: As shown in Figure 3, there were significant effects of EF performance group (low, medium, and high) in the Reading speed test, $F(2,133)=4.4, p<.05, \eta_{\mathrm{p}}{ }^{2}=.06$, and the Reading comprehension test, $F(2$, $133)=3.2, p<.05, \eta_{\mathrm{p}}{ }^{2}=.05$. In the Spelling test, there was a marginally significant effect of EF performance group, $F(2,130)=2.4, p=.09, \eta_{\mathrm{p}}{ }^{2}=.04$. Planned contrasts revealed that medium or high EF were associated with significantly increased performance in the Reading speed test (medium EF: $\eta_{\mathrm{p}}{ }^{2}=.03$; high EF: $\eta_{\mathrm{p}}{ }^{2}=.06$ ). For the Reading comprehension test and the Spelling test, only children with high EF differed significantly from children with low EF at T1 (Reading comprehension test: $\eta_{\mathrm{p}}{ }^{2}=.05$, Spelling test: $\eta_{\mathrm{p}}{ }^{2}=.04$ ).

Older cohort: There was a significant effect of EF performance group (low, medium and high) in the Reading speed test, $F(2,139)=3.4, p<.05, \eta_{\mathrm{p}}{ }^{2}=.05$, even after controlling for significant effects of the covariates of the nonverbal IQ, $F(1,139)=4.7, p<.05, \eta_{\mathrm{p}}{ }^{2}=.03$, and vocabulary skills, $F(1,139)=3.9, p<.05, \eta_{\mathrm{p}}{ }^{2}=.03$, and a marginal significant effect of the EF performance groups in the Reading comprehension test, $F(2,139)=2.9, p$ $=.06, \eta_{\mathrm{p}}{ }^{2}=.04$. For the Spelling test no significant effects of EF performance group, but a significant effect of the covariate SES, $F(1,130)=7.2, p<.01, \eta_{\mathrm{p}}{ }^{2}=.05$, was found. Planned contrasts revealed that medium or high EF were associated with significantly increased performance in the Reading speed test (medium EF: $\eta_{\mathrm{p}}{ }^{2}=.04$; high EF: $\eta_{\mathrm{p}}{ }^{2}=.04$ ), and in the Reading comprehension test (medium EF: $\eta_{\mathrm{p}}{ }^{2}=.03$; high EF: $\eta_{\mathrm{p}}{ }^{2}=.04$ ) at T3, compared to low EF at T1. 


\section{Discussion}

The present study adds some new results to the growing body of developmental and educational research supporting the key role of EF in young children's academic attainment and provides prospective longitudinal analyses of EF development from preschool to school age.

With respect to the developmental question of EF, the mixed ANOVA revealed significant main effects of time for all EF measures. These findings clearly demonstrate that preschool children continue to enhance their EF performance during their transition to school. Moreover, EF changes-measured with tasks tapping inhibition, working memory, and cognitive flexibility-were substantial; children improved their EF performance by one-half to three-quarters of a standard deviation in EF performance over any 12-month period. Interestingly, the magnitude of changes in EF was larger for the younger cohort compared to the older cohort, at least in two EF measures (inhibition, and working memory). Furthermore, subsequent analyses revealed that the improvement in EF performance was significantly larger for preschoolers (from pre-kindergarten to kindergarten) than school-age children (from grade 1 to grade 2), thus confirming our first hypothesis. These findings are in agreement with cross-sectional studies showing that early childhood is a time of pronounced EF development (e.g., Best et al., 2011; Davidson et al., 2006). The few longitudinal studies seem to support this hypothesis as well. Recently, similarly to our results, nonlinearity in the rate of EF development was shown in preschoolers-insofar as mean levels of changes in EF $(>1 S D)$ were larger for the younger than for the older children (aged 3 to 4 vs. 4 to 5 years; Willoughby et al., 2012b). Analogous, a pattern of nonlinear EF development has also been shown in school-age children-such as a lesser improvement in EF throughout grades 3 to 6 compared to earlier grades (i.e., grades 1 to 3, Altemeier et al., 2008). Collectively, these results document that developmental changes in EF, especially pronounced in preschool children, can hardly be explained by an artifact of different assessment methods, given the similarity in findings across studies. Additionally, profound changes in brain development (e.g.; changes in prefrontal-based circuitry), which are assumed to be related to EF development, seem to occur during this period as well, thus supporting the assumption that the preschool years are an important time for EF development (Bell \& Wolfe, 2007; Swingler, Willoughby, \& Calkins, 2011).

Although numerous tasks measuring EF in early childhood have been developed so far, they rarely demonstrate sufficient variability in performance to be used for an extended age range (Willoughby et al. 2012b). Therefore, a central challenge facing studies assessing EF development, is the selection of age-appropriate and age-sensitive tasks which are suitable for use over a longer period of time (e.g. from preschool to school years). Our selection of EF tasks seems to meet this demand insofar as neither floor nor ceiling effects were observed regarding EF performance in our sample of 5- to 8-year olds. Accordingly, it can be assumed that our tasks are complex enough to delineate individual differences in rapidly developing EF of preschool and young school age children. Furthermore, it can be proposed that our age-sensitive tasks allow the monitoring and evaluation of EF interventions during transition to school, an important point, given that there is evidence that $\mathrm{EF}$ trainings implemented in prekindergarten and kindergarten promote EF development (Thorell et al., 2008; Röthlisberger, Neuenschwander, Cimeli, Michel, \& Roebers, 2012).

The second purpose of this study was to determine the degree to which early EF are associated with different aspects of school achievement later on. Previous studies have predominantly focused on the concurrent relationship between EF and different school skills (e.g. Bull \& Sherif, 2001; Hooper et al., 2011; Sesma et al., 2009). The present study, however, with an extended length of duration, allowed us to more clearly qualify the predictive associations between EF and school achievement. Our hierarchical regression analyses indicate that EF measured in prekindergarten and kindergarten years do have substantial predictive power for mathematical, reading and spelling achievement two years later, when children are enrolled in first and second grade. Even when nonverbal IQ, vocabulary and SES were statistically controlled, EF continued to explain unique variances in nearly all academic skills (with one exception: Magnitude comparison test in second graders). As to the question of differential patterns of EF predicting academic skills, existing results are inconsistent. In our study, when the three preceding variables (SES, nonverbal IQ, vocabulary) were statistically accounted for, EF explained 6-20\% of unique variance in mathematic skills and 4-12\% of unique variance in spelling and reading tests, two years later. It could be assumed that the pattern of association between early EF and later academic achievement on the whole was quite similar for math, reading and spelling skills, suggesting a domain-general contribution of EF to academic performance. Additionally, our results suggest an intra-domain variability which is indicated by the results that EF was associated more highly with some tests than others within the same domain. As an example, kindergarten children's EF were more closely related to certain aspects of math (Sequences of numbers: early EF explained $20 \%$ of unique variances two years later) than others (Magnitude of comparison: early EF explained no additional variance two years later). One possible interpretation for this 
pattern of results may lie in the fact that EF may be more important in academic skills which are less automatic and require more effortful processing of information than others (Best et al., 2011). In generally, the current findings highlight the importance of early EF as a powerful predictor of academic functioning and suggest that, together with a domain specific approach focusing on emergent literacy and numeracy skills, EF should be considered when early education intervention and prevention of school failure are planned and applied.

The analysis of associations between EF and school achievement is informative in suggesting a developmental link between these skills (Welsh et al., 2010). In order to take an applied perspective, in which intervention or prevention programs are implemented, it should first be empirically established whether an early assessment of EF can provide sufficient information concerning poor EF as a risk factor for later mathematical, reading or spelling difficulties. This issue was examined in our study by comparing children with different EF performance levels at study entry. ANCOVAs demonstrated significant effects of EF performance group (low, medium, high) in various tests measuring mathematical and reading achievement two years later. In addition, planned contrasts revealed that low levels of EF, compared to medium and high levels of EF in preschool, were associated with one-half to one standard deviation disadvantage of mathematical or reading performance two years later in first and second grades. Considering that nonverbal IQ, vocabulary and SES were accounted for, the significant differences in academic performance of children identified as low EF performers are impressive. It may be speculated that children with higher levels of EF at school entry are more sensitive to math and reading instruction because of their ability to attend to and persist at a task, which could result in better academic skills. Additionally, it could be suggested, that children starting with low levels of EF, compared to children with medium and high EF, are struggling more when facing tasks with high-level cognitive demands (i.e., Sequences of numbers test) and that these skills may directly rely on EF to be executed. In sum, children with low EF in preschool years are more likely to show lower academic profiles in the first school years compared to children with medium and high EF. The findings also indicated that there is some variation in the magnitude of effects found across the different sub-tests within each academic domain. Possibly, part of variation was due to specifics of the subtests used in the present study.

\subsection{Conclusions}

To conclude, the present study extended previous empirical findings regarding developmental changes of $E F$ and the relationship between EF and academic achievement in 5- to 8-year-old children. Ongoing development was observed in the assessed EF, indicative of pronounced sensitivity of the applied tasks. The relationship between $\mathrm{EF}$ and academic achievement was subsequently illuminated by focusing on the predictive power of EF in different academic tasks on the one hand and on academic performance depending on different initial level of EF on the other hand. Given that EF continue to develop from preschool into school years, and that starting with low $\mathrm{EF}$ is associated with lower academic skills two years later, our findings pronounce the importance of considering EF performance when identifying children at risk for later academic difficulties. In combination with the assessment of children's emergent literacy or numeracy skills, they may give important implications for the direction of individualized remediation programs.

\section{Acknowledgements}

The present study was financially supported by the Jacobs Foundation through a grant to the senior author. We gratefully thank participating children, their parents, school administrators, teachers, and research staff who made this study possible.

\section{References}

Agostino, A., Johnson, J., \& Pascual-Leone, J. (2010). Executive functions underlying multiplicative reasoning: Problem type matters. Journal of Experimental Child Psychology, 105, 286-305. http://dx.doi.org/10.1016/j.jecp.2009.09.006 19913238

Altemeier, L. E., Abbott, R. D., \& Berninger, V. W. (2008). Executive functions for reading and writing in typical literacy development and dyslexia. Journal of Clinical and Experimental Neuropsychology, 30, 588-606. http://dx.doi.org/10.1080/13803390701562818 18569253

Archibald, S. J., \& Kerns, K. A. (1999). Identification and description of new tests of executive functioning in children. Child Neuropsychology, 5, 115-129.

Aunio, P., \& Niemivirta, M. (2010). Predicting children's mathematical performance in grade one by early numeracy. Learning and Individual Differences, 20, 427-435. http://dx.doi.org/10.1016/j.lindif.2010.06.003 
Bell, M. A., \& Wolfe, C. D. (2007). Changes in brain functioning from infancy to early childhood: Evidence from EEG power and coherence during working memory tasks. Developmental Neuropsychology, 31, 21-38. http://dx.doi.org/10.1207/s15326942dn3101_2

Best, J. R., \& Miller, P. H. (2010). A developmental perspective on executive function. [Peer Reviewed]. Child Development, 81, 1641-1660. http://dx.doi.org/10.1111/j.1467-8624.2010.01499.x 21077853

Best, J. R., Miller, P. H., \& Naglieri, J. A. (2011). Relations between executive function and academic achievement from ages 5 to 17 in a large, representative national sample. Learning and Individual Differences, 21, 327-336. http://dx.doi.org/10.1016/j.lindif.2011.01.007F 21845021

Blair, C., \& Razza, R. P. (2007). Relating effortful control, executive function, and false belief understanding to emerging math and literacy ability in kindergarten. Child Development, 78, 647-663. http://dx.doi.org/10.1111/j.1467-8624.2007.01019.x 17381795

Booth, J. N., Boyle, J. M. E., \& Kelly, S. W. (2010). Do tasks make a difference? Accounting for heterogeneity of performance of children with reading difficulties on tasks of executive function: Findings from a meta-analysis. [Peer Reviewed]. British Journal of Developmental Psychology, 28, 133-176. http://dx.doi.org/10.1348/026151009X485432

Borella, E., Carretti, B., \& Pelegrina, S. (2010). The specific role of inhibition in reading comprehension in good and poor comprehenders. [Peer Reviewed]. Journal of Learning Disabilities, 43, 541-552. http://dx.doi.org/10.1177/0022219410371676 20606207

Brock, L. L., Rimm-Kaufman, S. E., Nathanson, L., \& Grimm, K. J. (2009). The contributions of 'hot' and 'cool' executive function to children's academic achievement, learning-related behaviors, and engagement in kindergarten. [Peer Reviewed]. Early Childhood Research Quarterly, 24, 337-349. http://dx.doi.org/10.1016/j.ecresq.2009.06.001

Bull, R., \& Sherif, G. (2001). Executive functioning as a predictor of children's mathematical ability: Inhibition, switching, and working memory. Developmental Neuropsychology, 19, 273-293.

Bull, R., Espy, K. A., \& Wiebe, S. A. (2008). Short-term memory, working memory, and executive functioning in preschoolers: Longitudinal predictors of mathematical achievement at age 7 years. Developmental Neuropsychology, 33, 205-228. http://dx.doi.org/10.1080/87565640801982312 18473197

Bull, R., Espy, K. A., Wiebe, S. A., Sheffield, T. D., \& Nelson, J. M. (2011). Using confirmatory factor analysis to understand executive control in preschool children: Sources of variation in emergent mathematic achievement. Developmental Science, 14, 679-692. http://dx.doi.org/10.1111/j.1467-7687.2010.01012.x

Carlson, S. M. (2005). Developmentally sensitive measures of executive function in preschool children. Developmental Neuropsychology, 28, 595-616. http://dx.doi.org/10.1207/s15326942dn2802_3

Cattell, R. B., Weiß, R. H. \& Osterland, J. (1997). CFT 1: Grundintelligenztest Skala 1 (5., revid. Aufl.). Göttingen: Hogrefe

Christopher, M. E., Miyake, A., Keenan, J. M., Pennington, B., DeFries, J. C., Wadsworth, S. J., ... Olson, R. K. (2012). Predicting word reading and comprehension with executive function and speed measures across development: A latent variable analysis. Journal of Experimental Psychology: General, 141, 470-488. http://dx.doi.org/10.1037/a002737522352396

Clark, C. A. C., Pritchard, V. E., \& Woodward, L. J. (2010). Preschool executive functioning abilities predict early mathematics achievement. Developmental Psychology, 46, 1176-119. http://dx.doi.org/10.1037/a0019672 20822231

Davidson, M. C., Amso, D., Anderson, L. C., \& Diamond, A. (2006). Development of cognitive control and executive functions from 4 to 13 years: Evidence from manipulations of memory, inhibition, and task switching. Neuropsychologia, 44, 2037-2078. http://dx.doi.org/10.1016/j.neuropsychologia.2006.02.006 16580701

De Smedt, B., Verschaffel, L., \& Ghesquiere, P. (2009). The predictive value of numerical magnitude comparison for individual differences in mathematics achievement. Journal of Experimental Child Psychology, 103, 469-479. http://dx.doi.org/10.1016/j.jecp.2009.01.010

Diamond, A., Barnett, W. S., Thomas, J., \& Munro, S. (2007). Preschool program improves cognitive control. Nov 2007. Science, 318, 1387-1388. http://dx.doi.org/10.1126/science.1151148 18048670 
Duncan, G. J., Dowsett, C. J., Claessens, A., Magnuson, K., Huston, A. C., Klebanov, P., ... Japel, C. (2007). School readiness and later achievement. Developmental Psychology, 43, 1428-1446. http://dx.doi.org/10.1037/0012-1649.43.6.1428 18020822

Espy, K. A., McDiarmid, M. M., Cwik, M. F., Stalets, M. M., Hamby, A., \& Senn, T. E. (2004). The contribution of executive functions to emergent mathematic skills in preschool children. Developmental Neuropsychology, 26, 465-486. http://dx.doi.org/10.1207/s15326942dn2601_6

Garon, N., Bryson, S. E., \& Smith, I. M. (2008). Executive function in preschoolers: A review using an integrative framework. Psychological Bulletin, 134, 31-60. http://dx.doi.org/10.1037/0033-2909.134.1.31

Haffner, J., Baro, K., Parzer, P., \& Resch, F. (2005). Heidelberger Rechentest-HRT 1-4: Erfassung mathematischer Basiskompetenzen im Grundschulalter Göttingen, Germany: Hogrefe.

Hinshaw, S. P., Carte, E. T., Fan, C., Jassy, J. S., \& Owens, E. B. (2007). Neuropsychological functioning of girls with attention-deficit/hyperactivity disorder followed prospectively into adolescence: Evidence for continuing deficits? Neuropsychology, 21, 263-273. http://dx.doi.org/10.1037/0894-4105.21.2.263 17402826

Hooper, S. R., Costa, L.-J., McBee, M., Anderson, K. L., Yerby, D. C., Knuth, S. B., \& Childress, A. (2011). Concurrent and longitudinal neuropsychological contributors to written language expression in first and second grade students. Reading and Writing, 24, 221-252. http://dx.doi.org/10.1007/s11145-010-9263-x

Hughes, C., \& Ensor, R. (2007). Executive function and theory of mind: Predictive relations from ages 2 to 4. Developmental Psychology, 43, 1447-1459. http://dx.doi.org/10.1037/0012-1649.43.6.1447 18020823

Hughes, C., Ensor, R., Wilson, A., \& Graham, A. (2010). Tracking executive function across the transition to school: A latent variable approach. Developmental Neuropsychology, 35, 20-36. http://dx.doi.org/10.1080/87565640903325691 20390590

Jordan, N. C., Glutting, J., \& Ramineni, C. (2010). The importance of number sense to mathematics achievement in first and third grades. Learning and Individual Differences, 20, 82-88. http://dx.doi.org/10.1016/j.lindif.2009.07.004 20401327

Kaufmann, L., Nuerk, H.-C., Graf, M., Krinzinger, H., Delazer, M., \& Willmes, K. (2009). TEDI-MATH: Test zur Erfassung numerisch-rechnerischer Fertigkeiten vom Kindergarten bis zur 3. Klasse. Bern, Switzerland: Hans Huber.

Küspert, P., \& Schneider, W. (1998). Würzburger Leise Leseprobe (WLLP). Göttingen, Germany: Hogrefe.

Lee, K., Ng, S. F., Pe, M. L., Ang, S. Y., Hasshim, M. N. A. M., \& Bull, R. (2012). The cognitive underpinnings of emerging mathematical skills: Executive functioning, patterns, numeracy, and arithmetic. British Journal of Educational Psychology, 82, 82-99. http://dx.doi.org/10.1111/j.2044-8279.2010.02016.x

Lehto, J. E., Juujarvi, P., Kooistra, L., \& Pulkkinen, L. (2003). Dimensions of executive functioning: Evidence from children. British Journal of Developmental Psychology, 21, 59-80. http://dx.doi.org/10.1348/026151003321164627

Loher, S., \& Roebers, C. (2013). Executive Functions and Their Differential Contribution to Sustained Attention in 5- to 8-Year-Old Children. Journal of Educational and Developmental Psychology, 3, 51-63. http://dx.doi.org/10.5539/jedp.v3n1p51

May, P. (2002). Hamburger Schreib-Probe 1-9 (HSP). Hamburg, Germany: vpm.

Mayringer, H., \& Wimmer, H. (2005). Salzburger Lese-Screening für die Klassenstufen 1-4 (SLS) [Salzburg Reading Test (SLS). Bern, Switzerland: Hans Huber.

Michel, E., \& Roebers, C. M. (2008). Children in Regular and Special Needs Classes: Cognitive and

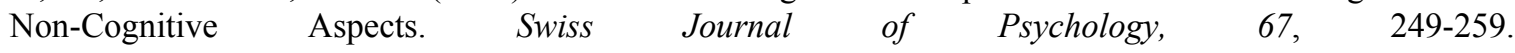
http://dx.doi.org/10.1024/1421-0185.67.4.249

Miyake, A., Friedman, N. P., Emerson, M. J., Witzki, A. H., Howerter, A., \& Wager, T. D. (2000). The unity and diversity of executive functions and their contributions to complex "Frontal Lobe" tasks: A latent variable analysis. Cognitive Psychology, 41, 49-100. http://dx.doi.org/10.1006/cogp.1999.0734

Monette, S., Bigras, M., \& Guay, M. C. (2011). The role of the executive functions in school achievement at the end of Grade 1. Journal of Experimental Child Psychology, 109, 158-173. http://dx.doi.org/10.1016/j.jecp.2011.01.008 21349537 
Navarro, J. I., Aguilar, M., Marchena, E., Ruiz, G., Menacho, I., \& Van Luit, J. E. H. (2012). Longitudinal study of low and high achievers in early mathematics. British Journal of Educational Psychology, 82, 28-41. http://dx.doi.org/10.1111/j.2044-8279.2011.02043.x

Pickering, S. J., \& Gathercole, S. E. (2001). Working Memory Test Battery for Children (WMTB-C). London: The Psychological Corporation.

Passolunghi, M. C., \& Lanfranchi, S. (2012). Domain-specific and domain-general precursors of mathematical achievement: A longitudinal study from kindergarten to first grade. British Journal of Educational Psychology, 82, 42-63. http://dx.doi.org/10.1111/j.2044-8279.2011.02039.x

Passolunghi, M. C., Vercelloni, B., \& Schadee, H. (2007). The precursors of mathematics learning: Working memory, phonological ability and numerical competence. [Peer Reviewed]. Cognitive Development, 22, 165-184. http://dx.doi.org/10.1016/j.cogdev.2006.09.001

Raghubar, K. P., Barnes, M. A., \& Hecht, S. A. (2010). Working memory and mathematics: A review of developmental, individual difference, and cognitive approaches. Learning and Individual Differences, 20, 110-122. http://dx.doi.org/10.1016/j.lindif.2009.10.005

Ricken, G., Fritz, A., Schuck, K.-D., \& Preuss, U. (2007). HAWIVA-III: Hannover-Wechsler-Intelligenztest für Kinder im Vorschulalter-III. Bern, Switzerland: Hans Huber.

Roebers, C. M., \& Kauer, M. (2009). Motor and cognitive control in a normative sample of 7-year-olds. Developmental Science, 12, 175-181. http://dx.doi.org/10.1111/j.1467-7687.2008.00755.x

Roebers, C. M., Röthlisberger, M., Cimeli, P., Michel, E., \& Neuenschwander, R. (2011). School enrolment and executive functioning: A longitudinal perspective on developmental changes, the influence of learning context, and the prediction of pre-academic skills. European Journal of Developmental Psychology, 8, 526-540. http://dx.doi.org/10.1080/17405629.2011.571841

Röthlisberger, M., Neuenschwander, R., Michel, E., \& Roebers, C. M. (2010). Exekutive Funktionen: Zugrundeliegende kognitive Prozesse und deren Korrelate bei Kindern im späten Vorschulalter [Executive Functions: Underlying Cognitive Processes and Their Correlates in Late Preschool Children]. Zeitschrift für $\begin{array}{llll}\text { Entwicklungspsychologie } \quad \text { und Pädagogische } & \text { Psychologie, }\end{array}$ http://dx.doi.org/10.1026/0049-8637/a000010

Röthlisberger, M., Neuenschwander, R., Cimeli, P., Michel, E., \& Roebers, C. M. (2012). Improving executive functions in 5- and 6-year-olds: Evaluation of a small group intervention in prekindergarten and kindergarten children. Infant and Child Development, 21, 411-429. http://dx.doi.org/10.1002/Icd.752

Schmid, C., Zoelch, C., \& Roebers, C. M. (2008). Working memory in 4- to 5-year-old children: Theoretical issues and empirical findings. Zeitschrift für Entwicklungspsychologie und Pädagogische Psychologie, 40, 2-12. http://dx.doi.org/10.1026/0049-8637.40.1.2

Sesma, H. W., Mahone, E. M., Levine, T., Eason, S. H., \& Cutting, L. E. (2009). The contribution of executive skills to reading comprehension. Child Neuropsychology, 15, 232-246. http://dx.doi.org/10.1080/09297040802220029 18629674

Schneider, W. (2009). The development of reading and spelling: Relevant precursors, developmental changes, and individual differences. In W. Schneider, \& M. Bullock (Eds.), Human development from early childhood to early adulthood. Findings from a 20 year longitudinal study (pp. 199-220). New York: Psychology Press.

Swingler, M. M., Willoughby, M. T., \& Calkins, S. D. (2011). EEG Power and Coherence During Preschoolers' Performance of an Executive Function Battery. Developmental Psychobiology, 53, 771-784. http://dx.doi.org/10.1002/Dev.20588

Thorell, L. B., Lindqvist, S., Nutley, S. B., Bohlin, G., \& Klingberg, T. (2009). Training and transfer effects of executive functions in preschool children. Developmental Science, 12, 106-113. http://dx.doi.org/10.1111/j.1467-7687.2008.00745.x 19120418

Toll, S. W., Van der Ven, S. H., Kroesbergen, E. H., \& Van Luit, J. E. (2011). Executive functions as predictors of math learning disabilities. Journal of Learning Disabilities, 44, 521-532. http://dx.doi.org/10.1177/0022219410387302 
Van der Sluis, S., de Jong, P. F., \& van der Leij, A. (2007). Executive functioning in children, and its relations with reasoning, reading, and arithmetic. Intelligence, 35, 427-449. http://dx.doi.org/10.1016/j.intell.2006.09.001

Van der Ven, S. H. G., Kroesbergen, E. H., Boom, J., \& Leseman, P. P. M. (2012). The development of executive functions and early mathematics: A dynamic relationship. British Journal of Educational Psychology, 82, 100-119. http://dx.doi.org/10.1111/j.2044-8279.2011.02035.x

Wegener, B. (1988). Kritik des Prestiges. Opladen: Westdeutscher Verlag.

Welsh, J. A., Nix, R. L., Blair, C., Bierman, K. L., \& Nelson, K. E. (2010). The development of cognitive skills and gains in academic school readiness for children from low-income families. Journal of Educational Psychology, 102, 43-53. http://dx.doi.org/10.1037/a0016738 20411025

Wiebe, S. A., Espy, K. A., \& Charak, D. (2008). Using confirmatory factor analysis to understand executive control in preschool children: I. Latent structure. Developmental Psychology, 44, 575-587. http://dx.doi.org/10.1037/0012-1649.44.2.575 18331145

Wiebe, S. A., Sheffield, T., Nelson, J. M., Clark, C. A. C., Chevalier, N., \& Espy, K. A. (2011). The structure of executive function in 3-year-olds. [Peer Reviewed]. Journal of Experimental Child Psychology, 108, 436-452. http://dx.doi.org/10.1016/j.jecp.2010.08.008 20884004

Willoughby, M. T., Blair, C. B., Wirth, R. J., \& Greenberg, M. (2012a). The measurement of executive function at age 5: Psychometric properties and relationship to academic achievement. Psychological Assessment, 24, 226-239. http://dx.doi.org/10.1037/a0025361 21966934

Willoughby, M. T., Wirth, R. J., Blair, C. B., \& Investigators, F. L. P. (2012b). Executive Function in Early Childhood: Longitudinal Measurement Invariance and Developmental Change. Psychological Assessment, 24, 418-431. http://dx.doi.org/10.1037/A0025779

Zimmermann, P., Gondan, M., \& Fimm, B. (2004). Testbatterie zur Aufmerksamkeitsprüfung für Kinder (KITAP). Herzogenrath, Germany: Psytest.

\section{Copyrights}

Copyright for this article is retained by the author(s), with first publication rights granted to the journal.

This is an open-access article distributed under the terms and conditions of the Creative Commons Attribution license (http://creativecommons.org/licenses/by/3.0/). 